\title{
FLUXOS DE ÓXIDO NITROSO EM SOLOS COM COBERTURA DE FLORESTA OMBRÓFILA DENSA MONTANA NA SERRA DOS ÓRGÃOS, RIO DE JANEIRO
}

\author{
Renato de Aragão Ribeiro Rodrigues \\ Empresa Brasileira de Pesquisa Agropecuária, Embrapa Agrossilvipastoril, CP 343, 78550-970 Sinop - MT, Brasil \\ William Zamboni de Mello* \\ Departamento de Geoquímica, Instituto de Química, Universidade Federal Fluminense, Outeiro de São João Batista, s/n, \\ 24020-007 Niterói - RJ, Brasil
}

Recebido em 18/11/11; aceito em 3/4/12; publicado na web em 3/7/12

\begin{abstract}
NITROUS OXIDE FLUXES IN MONTANE TROPICAL RAINFOREST SOIL IN SERRA DOS ÓRGÃOS, RJ. Soil fluxes of $\mathrm{N}_{2} \mathrm{O}$ were determined over one year in montane tropical rainforest of southeastern Brazil with average annual rainfall of $2.8 \mathrm{~m}$. Annual mean ( \pm standard deviation) and median $\mathrm{N}_{2} \mathrm{O}$ fluxes were $3.0 \pm 1.4$ and $2.7 \mu \mathrm{g} \mathrm{N} \mathrm{m} \mathrm{m}^{-2} \mathrm{~h}^{-1}$, respectively, is 5-10 times lower than mean values reported in literature for tropical rainforest soils in the Amazon basin. $\mathrm{N}_{2} \mathrm{O}$ fluxes varied spatially and seasonally, were about twice as high during summer as in winter, and significantly influenced by both monthly precipitation and soil temperature.
\end{abstract}

Keywords: soil gas fluxes; nitrogen biogeochemical cycle; ozone-depleting substances.

\section{INTRODUÇÃO}

$\mathrm{O}$ óxido nitroso $\left(\mathrm{N}_{2} \mathrm{O}\right)$ exerce importante papel no controle de processos físicos e químicos da atmosfera, é naturalmente um dos principais gases do efeito estufa e uma das substâncias responsáveis pelo consumo do ozônio $\left(\mathrm{O}_{3}\right)$ na estratosfera. Possui um potencial de aquecimento global aproximadamente 300 vezes superior ao do dióxido de carbono $\left(\mathrm{CO}_{2}\right)$. Por ser um gás inerte na troposfera, permanece na atmosfera por aproximadamente 120 anos até que se transforme, na estratosfera, em nitrogênio diatômico $\left(\mathrm{N}_{2}\right)$ e óxido nítrico (NO) por fotólise e reação com átomos de oxigênio atômico eletronicamente excitado $\left[\mathrm{O}\left({ }^{1} \mathrm{D}\right)\right] .{ }^{1} \mathrm{O}$ NO formado desencadeia, então, um processo cíclico de destruição do $\mathrm{O}_{3}$ estratosférico. ${ }^{1} \mathrm{O}$ aumento dos níveis estratosféricos de $\mathrm{NO}$, atribuído às emissões antrópicas de $\mathrm{N}_{2} \mathrm{O}$, resulta em declínio da camada de $\mathrm{O}_{3}$ estratosférica e, consequentemente, maior incidência da radiação UV-B (280-320 nm; n $=10^{-9}$ ) sobre a superfície da Terra. ${ }^{2}$ Face à redução das emissões dos clorofluorcarbonos, ${ }^{3,4}$ atribuída ao Protocolo de Montreal assinado em 1987, o $\mathrm{N}_{2} \mathrm{O}$ deverá se tornar a principal substância de origem antrópica responsável pelo consumo do $\mathrm{O}_{3}$ estratosférico. ${ }^{5}$

Há aproximadamente dois séculos e meio, as concentrações de $\mathrm{N}_{2} \mathrm{O}$ na atmosfera iniciaram um processo de ascensão contínuo além da faixa (260-275 ppb; ppb = parte por bilhão em volume) na qual oscilou ao longo dos últimos 1000 anos. ${ }^{6}$ Atualmente, a concentração atmosférica do $\mathrm{N}_{2} \mathrm{O}$ encontra-se próxima a 324 ppb. ${ }^{3,4}$ Esse crescimento é atribuído principalmente ao aumento das atividades agrícolas e pecuária para atender À demanda global por alimentos. ${ }^{6}$ No solo, a produção de $\mathrm{N}_{2} \mathrm{O}$ decorre dos processos microbiológicos de nitrificação e desnitrificação, sendo estimulada pela adição de fertilizantes nitrogenados e aporte de excretas dos rebanhos.

Os solos são as principais fontes globais de $\mathrm{N}_{2} \mathrm{O}$, cuja emissão é estimada em $c a$. $10 \mathrm{Tg} \mathrm{N}$ ano $^{-1}$, dos quais $c a$. $40 \%$ provêm de solos agrícolas e o restante de solos naturais. ${ }^{6}$ Os solos com cobertura de florestas tropicais pluviais são as principais fontes naturais globais de $\mathrm{N}_{2} \mathrm{O}$, com contribuição estimada de $c a$. 3,0 Tg N ano ${ }^{-1} .{ }^{6}$ Nos solos tropicais, a rápida mineralização da matéria orgânica, estimulada pela umidade e temperaturas elevadas, oferece condições favoráveis

*e-mail: zamboni@geoq.uff.br à produção microbiológica do $\mathrm{N}_{2} \mathrm{O}$ quando comparada aos solos das regiões de clima temperado. ${ }^{7,8} \mathrm{O}$ Brasil detém a maior área de florestas tropicais do mundo. O Bioma Amazônia ocupava originalmente uma área equivalente a $8,2 \times 10^{8}$ ha na América do Sul, dos quais ca. 50\% dentro do território brasileiro. ${ }^{9} \mathrm{O}$ Bioma Mata Atlântica, o terceiro maior do Brasil, ocupava $1,3 \times 10^{8}$ ha do território nacional. ${ }^{10}$ Dos vários estudos voltados às emissões de $\mathrm{N}_{2} \mathrm{O}$ em solos com cobetura de Floresta Ombrófila Densa (Floresta Pluvial Tropical) no Brasil, poucos foram realizados no Bioma Mata Atlântica, ${ }^{11-14} \mathrm{e}$ menos ainda na faixa altitudinal de formação Montana, ${ }^{13,14}$ ou seja, aquela que se situa entre 500 e $1.500 \mathrm{~m}$ do trecho da Mata Atlântica limitado à faixa latitudinal $16-24^{\circ} \mathrm{S} .{ }^{15}$

$\mathrm{O}$ objetivo deste trabalho foi estimar as emissões de $\mathrm{N}_{2} \mathrm{O}$ na interface solo-atmosfera numa área com cobertura de Floresta Ombrófila Densa Montana e verificar a influência da pluviosidade e temperatura do solo como fatores reguladores das variações sazonais das emissões de $\mathrm{N}_{2} \mathrm{O}$. A área estudada situa-se dentro dos limites de uma unidade de conservação federal na Serra dos Órgãos, Região Serrana do Rio de Janeiro. Esta área é potencialmente sujeita aos impactos provocados pelas deposições atmosféricas de poluentes oriundos da Região Metropolitana do Rio de Janeiro face à proximidade entre estes domínios e a diversas características geográficas regionais, tais como, clima, relevo, urbanização e industrialização. ${ }^{16,17}$

\section{PARTE EXPERIMENTAL}

\section{Área de estudo}

O estudo foi realizado numa área da Serra dos Órgãos com cobertura de Floresta Ombrófila Densa Montana, situada dentro dos limites do Parque Nacional da Serra dos Órgãos (PARNASO), no estado do Rio de Janeiro (RJ) (Figura 1S, material suplementar). A Serra dos Órgãos, cujo ponto culminante é a Pedra do Sino (2.263 $\mathrm{m})$, é o nome local da Serra do Mar, cujas vertentes íngremes voltadas para o Atlântico formam o limite norte da bacia hidrográfica da Baía de Guanabara. A Serra dos Órgãos possui coberturas de vegetação que variam, em função da altitude, de Floresta Ombrófila Densa Submontana até Campos de Altitude. No PARNASO, a Floresta 
Ombrófila Densa Montana abrange uma área de 2.303 ha, o que representa $21,5 \%$ da área total daquela unidade de conservação. ${ }^{18}$

As partes mais elevadas da Serra dos Órgãos, bem como o maciço do Itatiaia (Serra da Mantiqueira), registram as temperaturas mais baixas do RJ. Nas proximidades da Sede de Teresópolis do PARNASO, situada a $\mathrm{ca}$. $1.000 \mathrm{~m}$ de altitude (latitude 22\%26'58" S e longitude $42^{\circ} 59^{\prime} 07^{\prime}$ ' W), as médias mensais de longo termo de temperatura variam de $14,3{ }^{\circ} \mathrm{C}$ (julho) a $20,9^{\circ} \mathrm{C}$ (fevereiro) e no caso da precipitação, de $88 \mathrm{~mm}$ (junho) a $408 \mathrm{~mm}$ (dezembro). ${ }^{19}$ As médias anuais de temperatura e precipitação são $17,6^{\circ} \mathrm{Ce} 2.813 \mathrm{~mm} .{ }^{19} \mathrm{Cerca}$ de $70 \%$ da precipitação anual concentra-se no período de outubro a março. Nas altitudes mais elevadas, a temperatura atinge valores inferiores a $0{ }^{\circ} \mathrm{C}$ nos dias de inverno e a precipitação anual pode atingir até $3.600 \mathrm{~mm}$. Devido à proximidade com o mar (poucas dezenas de quilômetros), a serra se torna uma barreira para a entrada das massas de ar úmidas vindas do Atlântico. Em virtude disso, as chuvas orográficas sobre as vertentes voltadas para o Atlântico fazem com que estas sejam mais úmidas que aquelas voltadas para o interior do continente, i.e., as que compreendem o vale do rio Paraíba do Sul.

\section{Coleta de amostras}

As medidas de fluxos de $\mathrm{N}_{2} \mathrm{O}$ na interface solo-atmosfera foram realizadas no período de janeiro a dezembro de 2005 . Foram efetuadas 19 campanhas e amostrados 7 pontos distribuídos ao longo da trilha Mozart Catão (extensão: $c a$. 800 m; altitude: 1100-1200 m), situada no extremo nordeste do PARNASO. Este local situa-se, na direção N-NE, a 55-60 km dos centros dos municípios de Niterói e do Rio de Janeiro e, na direção N-NW, a $c a$. 28 km do centro do Complexo Petroquímico do Rio de Janeiro (COMPERJ).

As coletas de amostras foram realizadas sempre pela manhã, em geral, no período entre 10 e $12 \mathrm{~h}$. Os fluxos foram medidos por meio de câmaras de PVC, com $30 \mathrm{~cm}$ de diâmetro e $15 \mathrm{~cm}$ de altura. Para medidas dos fluxos, as câmaras foram inseridas $c a .2 \mathrm{~cm}$ de profundidade da superfície do solo (Figura 1). A inserção da câmara foi feita através de movimentos giratórios, de forma a minimizar alterações na serrapilheira e, ao mesmo tempo, assegurar boa vedação. Imediatamente após a inserção da câmara, uma amostra de ar atmosférico $\left(\mathrm{ca} .20 \mathrm{~cm}^{3}\right.$ ) foi recolhida em seringa de plástico de $60 \mathrm{~cm}^{3}$. Dez minutos após a inserção da câmara, uma primeira amostra de ar foi retirada de seu interior, seguida de outras 4 amostras retiradas em intervalos de $10 \mathrm{~min}$. As seringas utilizadas tinham acopladas válvulas plásticas de três vias, que asseguravam sua vedação após a

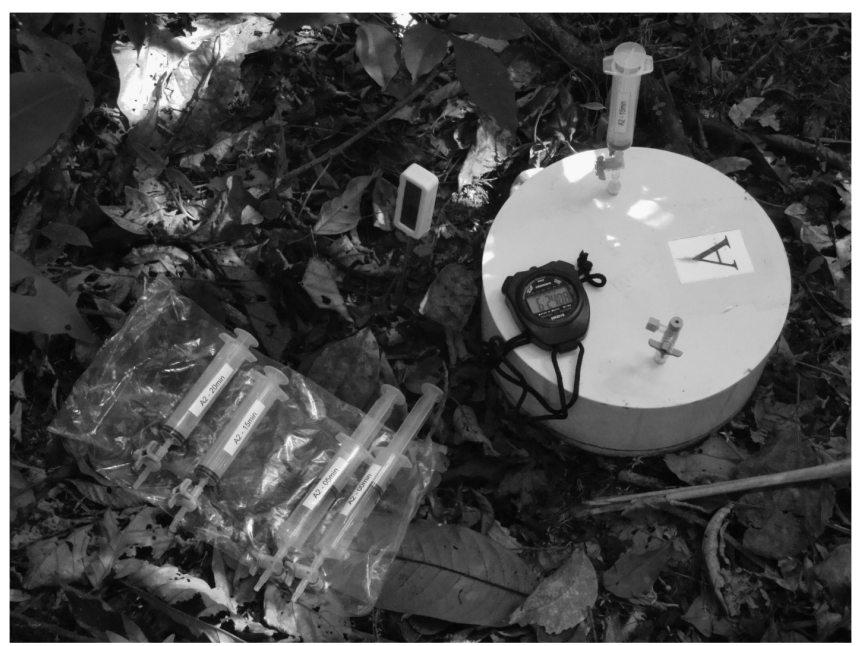

Figura 1. Câmara utilizada para medição do fluxo de óxido nitroso na interface solo-atmosfera coleta das amostras. Durante a amostragem foram medidas as temperaturas do ar e do solo.

Após cada coleta, as seringas foram transportadas para o Laboratório de Biogeoquímica de Ambientes Tropicais do Departamento de Geoquímica da UFF. As determinações de $\mathrm{N}_{2} \mathrm{O}$ foram efetuadas no período da tarde dos mesmos dias das coletas.

\section{Determinação de óxido nitroso}

A quantificação do $\mathrm{N}_{2} \mathrm{O}$ foi efetuada num cromatógrafo a gás Shimadzu modelo GC-17 equipado com detector de captura de elétrons de ${ }^{63} \mathrm{Ni}$. O gás de arraste utilizado, a uma vazão de 30 $\mathrm{cm}^{3} \mathrm{~min}^{-1}$, foi argônio contendo 5\% de metano (White Martins). O volume de amostra injetado, através de um loop fixado a uma válvula em aço inox de 6 vias, foi de $2 \mathrm{~cm}^{3}$. A coluna cromatográfica, em aço inox e empacotada com Porapak Q (80-100 mesh), possui $4 \mathrm{~m}$ de comprimento e 1/8 de polegada de diâmetro. Durante as análises, as temperaturas do detector e da coluna cromatográfica foram mantidas a 340 e $60{ }^{\circ} \mathrm{C}$, respectivamente. Para quantificação das concentrações de $\mathrm{N}_{2} \mathrm{O}$ nas amostras foram utilizados padrões de 356 e 840 ppb (White Martins, Gases Especiais). Os resultados foram registrados por meio do software Shimadzu CLASS CR-10, executado em MS-Windows.

O fluxo $(F)$ de $\mathrm{N}_{2} \mathrm{O}$ na interface solo-atmosfera foi calculado utilizando a Equação 1:

$$
F=205(P / T) h[d C / d t]_{t=0}
$$

onde 205 é o fator de ajuste de unidade para o $F$ expresso em $\mu \mathrm{g} \mathrm{N} \mathrm{m}{ }^{-2} \mathrm{~h}^{-1}, P$ a pressão atmosférica (em atm), $T$ a temperatura do ar $($ em K $), h$ a altura da câmara (em cm) e $[d C / d t]_{t=0}$ a inclinação da curva de variação de concentração de $\mathrm{N}_{2} \mathrm{O}$ (em ppb) em função do tempo (em min) em $t=0 \cdot{ }^{8,20}$ A pressão atmosférica utilizada na Equação 1 foi 0,87 atm, valor médio correspondente à altitude da área de estudo.

\section{RESULTADOS E DISCUSSÃO}

O fluxo médio de $\mathrm{N}_{2} \mathrm{O}$ (média aritmética \pm desvio padrão) foi $3,0 \pm 1,4 \mu \mathrm{g} \mathrm{N} \mathrm{m}^{-2} \mathrm{~h}^{-1}$ (mediana $=2,7 \mu \mathrm{g} \mathrm{N} \mathrm{m}^{-2} \mathrm{~h}^{-1}$ ) e as médias por campanha $(\mathrm{n}=19$ campanhas) variaram de $1,7 \pm 0,7$ a 4,6 $\pm 1,6 \mu \mathrm{g}$ $\mathrm{N} \mathrm{m}^{-2} \mathrm{~h}^{-1}$. O valor mais elevado de fluxo das medições individuais (por câmara) foi $8,2 \mu \mathrm{g} \mathrm{N} \mathrm{m}{ }^{-2} \mathrm{~h}^{-1}$ e o menor $-1,6 \mu \mathrm{g} \mathrm{N} \mathrm{m} \mathrm{N}^{-2} \mathrm{~h}^{-1}$, sendo este último o único resultado com sinal negativo obtido em um total de 125 medidas de fluxos realizadas. O sinal negativo denota transferência do gás da atmosfera para o solo, ${ }^{21}$ geralmente interpretado como consumo do $\mathrm{N}_{2} \mathrm{O}$ atmosférico pela desnitrificação, processo microbiológico que reduz o $\mathrm{N}_{2} \mathrm{O}$ a nitrogênio diatômico $\left(\mathrm{N}_{2}\right)$. Os fluxos médios por ponto de amostragem variaram de $2,4 \pm 1,3$ a 3,9 $\pm 1,9 \mu \mathrm{g} \mathrm{N} \mathrm{m}{ }^{-2} \mathrm{~h}^{-1}$, ou seja, ao longo do ano a variação espacial foi inferior à variação temporal.

Os fluxos de $\mathrm{N}_{2} \mathrm{O}$ variaram sazonalmente, com os menores valores no período de maio a julho e os maiores de setembro a janeiro (Figura 2). Portanto, os menores fluxos se deram no inverno e os maiores, durante a primavera e primeira metade do verão. Comportamento sazonal semelhante foi reportado, em altitude idêntica, por Souza Neto et al. ${ }^{13} \mathrm{em}$ um trecho da Serra do Mar, situado ao norte de Ubatuba (SP), onde os menores fluxos de $\mathrm{N}_{2} \mathrm{O}$ ocorreram no período de julho a setembro e os maiores em janeiro.

$\mathrm{Na}$ Serra dos Órgãos, os fluxos de $\mathrm{N}_{2} \mathrm{O}$ apresentaram uma diminuição gradual da segunda metade do verão até o início do inverno, mostrando pouca variação nesse período, mas subiram abruptamente de agosto a setembro, quando a emissão média se elevou de 2,2 para $4,0 \mu \mathrm{g} \mathrm{N} \mathrm{m}^{-2} \mathrm{~h}^{-2}$ (Figura 2). Comportamento semelhante foi verificado 


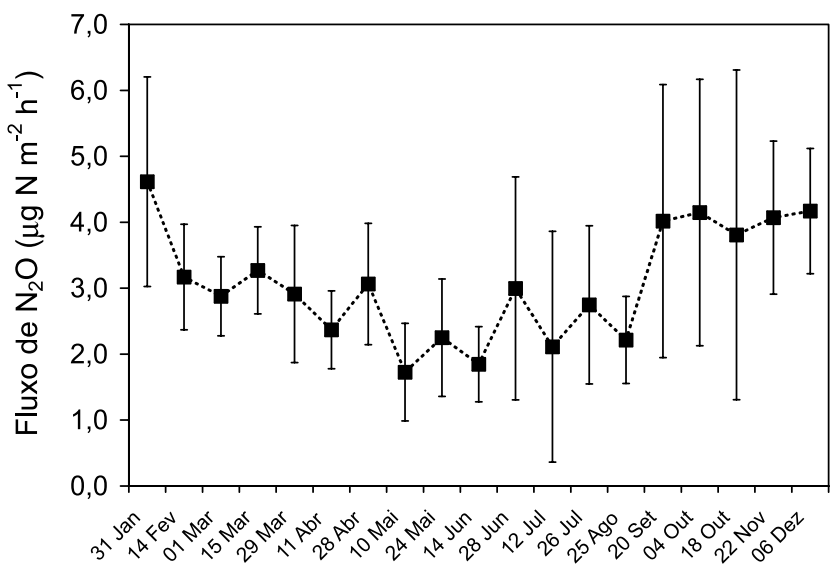

Figura 2. Variação temporal das emissões de óxido nitroso em solo com cobertura de Floresta Ombrófila Densa Montana na Serra dos Órgãos (média aritmética e desvio padrão representados por quadrado e barras; $n=7$, exceto janeiro quando $n=4$ )

no estudo realizado ao longo de 24 meses por $\operatorname{Costa}^{14}$ no Maciço da Tijuca (RJ), onde no primeiro ano o fluxo médio de $\mathrm{N}_{2} \mathrm{O}$ cresceu de 6,8 para $32 \mu \mathrm{g} \mathrm{N} \mathrm{m}^{-2} \mathrm{~h}^{-1}$ de agosto a setembro e, no segundo ano consecutivo, nesse mesmo período, de 6,6 para $46 \mu \mathrm{g} \mathrm{N} \mathrm{m}^{-2} \mathrm{~h}^{-1}$. No caso da Serra dos Órgãos, a elevação abrupta nas emissões de $\mathrm{N}_{2} \mathrm{O}$ neste período do ano pode estar associada, pelo menos parcialmente, à fase de transição entre o período de estiagem e o das chuvas na região, bem marcada, no ano de estudo (2005), pela acentuada diferença em quantidade de chuva precipitada no mês de setembro $(177 \mathrm{~mm})$ em relação à de agosto $(20 \mathrm{~mm})$ quando comparadas às médias mensais de longo termo que são, para esses meses, $140 \mathrm{e}$ $112 \mathrm{~mm}$, respectivamente. ${ }^{19}$

A Figura 3a mostra as variações dos fluxos médios mensais de $\mathrm{N}_{2} \mathrm{O}$ e da precipitação acumulada mensal de janeiro a dezembro. Nesse caso, foram utilizadas as médias aritméticas mensais para os meses em que houve mais de uma campanha. A influência da precipitação sobre os fluxos de $\mathrm{N}_{2} \mathrm{O}$ é validada pela correlação linear positiva e estatisticamente significativa entre os fluxos médios mensais de $\mathrm{N}_{2} \mathrm{O}$ e as precipitações acumuladas mensais $\left(\mathrm{r}^{2}=0,60 ; \mathrm{P}<0,01 ; \mathrm{n}=12\right.$ meses) (Figura 3b). O mesmo se verificou também, porém com um coeficiente de determinação inferior, entre os fluxos médios diários de $\mathrm{N}_{2} \mathrm{O}$ e as precipitações acumuladas semanalmente $\left(\mathrm{r}^{2}=0,35 ; \mathrm{P}\right.$ $<0,01 ; \mathrm{n}=19$ campanhas). Essa diferença se deve à atenuação da variabilidade quando do uso das médias mensais, favorecendo o aumento do coeficiente de determinação. Não se dispõe de dados de precipitação diários para o período estudado, uma vez que as precipitações acumuladas foram obtidas semanalmente de um coletor automático de chuva destinado à coleta de amostras para determinação da composição química da água de chuva. ${ }^{17}$ Em um estudo realizado num trecho da Floresta Ombrófila Densa Submontana na Reserva Biológica de Tinguá, Maddock et al. ${ }^{12}$ destacaram a forte influência positiva das chuvas sobre as emissões de $\mathrm{N}_{2} \mathrm{O}$ do solo, relatando aumento das emissões de até uma ordem de grandeza durante ou imediatamente após um evento de chuva.

A influência das chuvas sobre os fluxos de $\mathrm{N}_{2} \mathrm{O}$ está relacionada ao aumento da umidade e do aporte de nitrogênio no solo. $\mathrm{O}$ nitrato, amônio e ureia ${ }^{22}$ oriundos da lavagem do dossel da floresta, somados àquele já presente na água da chuva, são substratos para as bactérias nitrificantes e desnitrificantes no solo. ${ }^{23} \mathrm{~A}$ umidade, juntamente com a textura (ou granulometria) do solo, controla a taxa de difusão do oxigênio $\left(\mathrm{O}_{2}\right)$ atmosférico no solo. $\mathrm{O}_{2}$ é necessário aos processos microbiológicos responsáveis pela decomposição da matéria orgânica e nitrificação, sendo este último um dos processos
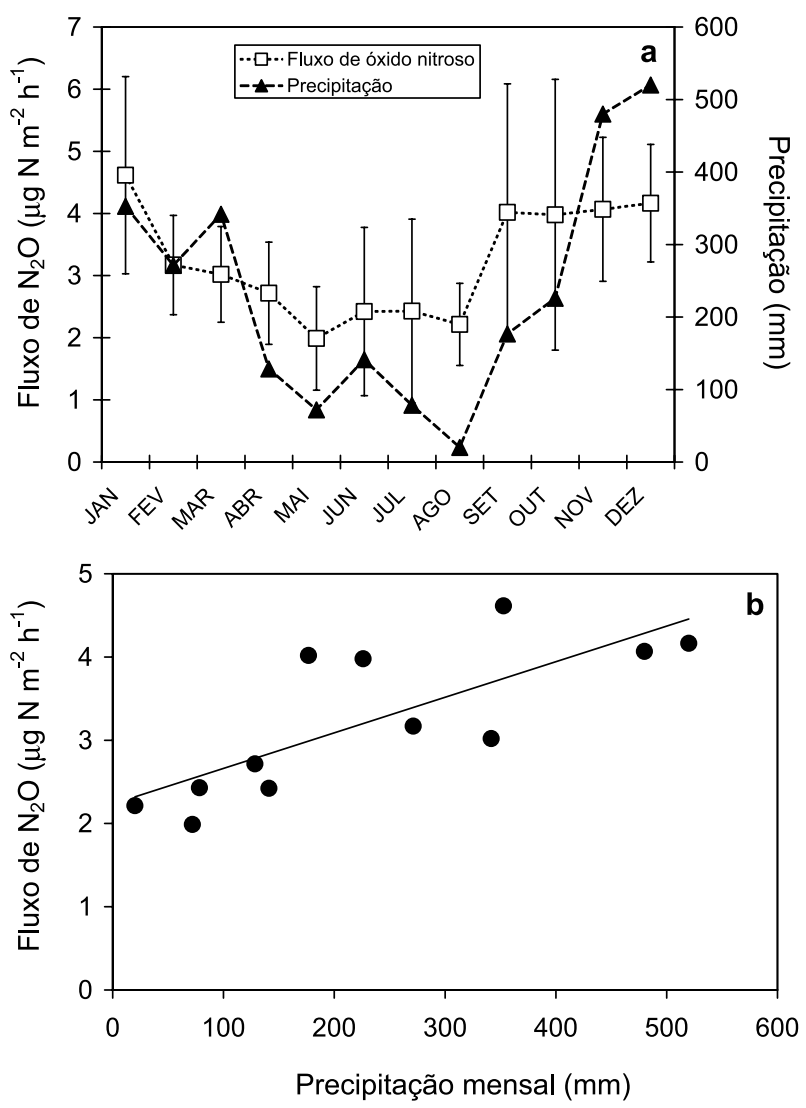

Figura 3. (a) Variação mensal dos fluxos de óxido nitroso (média aritmética \pm desvio padrão) e das precipitações acumuladas mensais. (b) Correlação entre os fluxos de óxido nitroso e as precipitações acumuladas mensais

pelo qual se forma o $\mathrm{N}_{2} \mathrm{O}$ a partir da etapa de oxidação do amônio a nitrito nos solos. ${ }^{23} \mathrm{~A}$ presença do $\mathrm{O}_{2}$ inibe a desnitrificação, mas na ausência dele, provocada pelo excesso de umidade no solo, este mesmo processo constitui uma outra fonte de produção de $\mathrm{N}_{2} \mathrm{O} \cdot{ }^{23} \mathrm{O}$ excesso de umidade no solo pode também impedir a transferência do $\mathrm{N}_{2} \mathrm{O}$ para a atmosfera, tornando-o, nesse caso, sujeito à redução a $\mathrm{N}_{2}$ pela desnitrificação no solo..$^{23}$

A temperatura do solo durante as medições de fluxos de $\mathrm{N}_{2} \mathrm{O}$, efetuadas sempre no período da manhã entre 10 e $12 \mathrm{~h}$, variou de 13 a $19^{\circ} \mathrm{C}$, com a mínima verificada em junho e a máxima nos meses de outubro a março. A Figura 4a mostra as variações dos fluxos médios mensais de $\mathrm{N}_{2} \mathrm{O}$ e da temperatura do solo, medidos simultaneamente, no período estudado. Da mesma forma que a precipitação, a temperatura parece exercer influência significativa sobre os fluxos de $\mathrm{N}_{2} \mathrm{O}$, corroborada pela correlação linear positiva e estatisticamente significativa entre os fluxos médios mensais de $\mathrm{N}_{2} \mathrm{O}$ e as temperaturas do solo $\left(\mathrm{r}^{2}=0,57 ; \mathrm{P}<0,01 ; \mathrm{n}=12\right.$ ) (Figura $\left.4 \mathrm{~b}\right)$. Uma correlação linear estatisticamente significativa foi verificada também entre os fluxos médios diários de $\mathrm{N}_{2} \mathrm{O}$ e a temperatura do solo $\left(\mathrm{r}^{2}=0,44 ; \mathrm{P}\right.$ $<0,01 ; \mathrm{n}=19)$.

O fluxo médio anual de $\mathrm{N}_{2} \mathrm{O}$ no trecho de altitude intermediária de domínio da Floresta Ombrófila Densa Montana na Serra dos Órgãos determinado neste estudo $\left(0,26 \mathrm{~kg} \mathrm{~N} \mathrm{ha}^{-1} \mathrm{ano}^{-1}\right)$ é o menor em relação aos reportados em outros estudos realizados na Mata Atlântica e Floresta Amazônica (Tabela 1). O fluxo de $\mathrm{N}_{2} \mathrm{O}$ medido na Serra dos Órgãos é 3 vezes menor que o fluxo médio encontrado por Souza Neto et al..$^{13}$ para solo com cobertura da Floresta Ombrófila Densa Montana, no Parque Estadual da Serra do Mar (SP). Estudos realizados em altitudes inferiores, compreendendo o trecho de Floresta Ombrófila Densa Submontana da Mata Atlântica, mostram fluxos de 

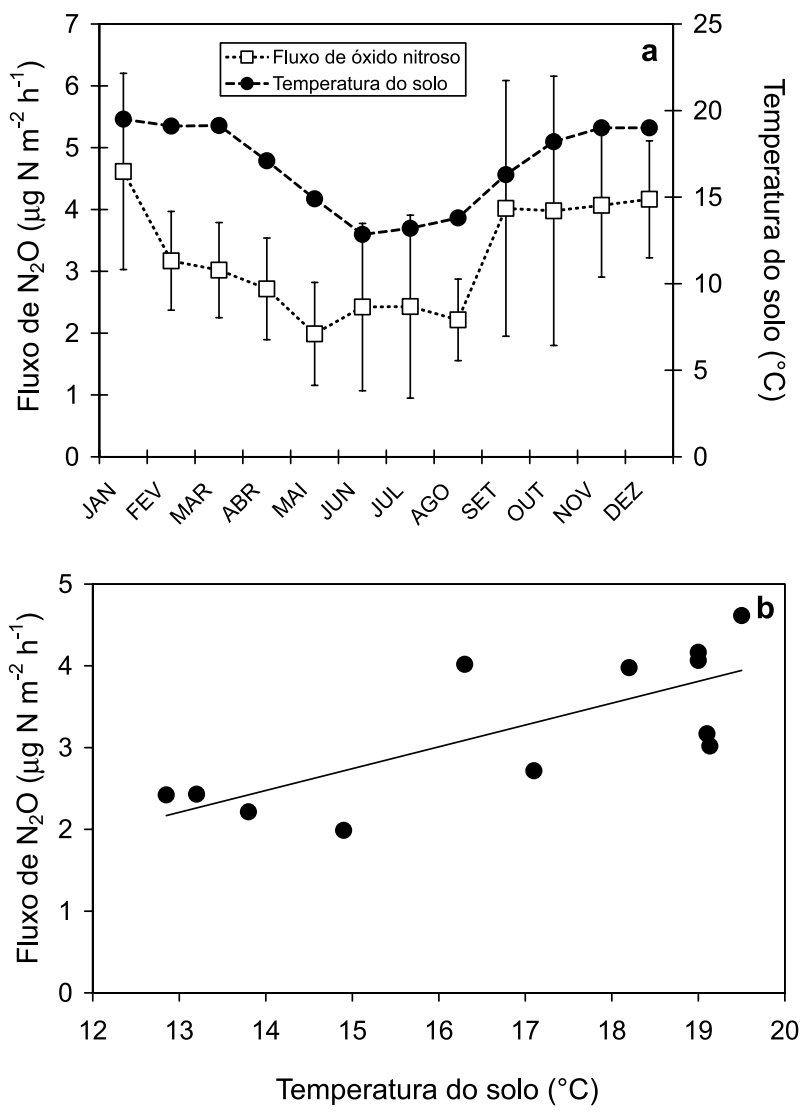

Figura 4. (a) Variação mensal dos fluxos de óxido nitroso (média aritmética \pm desvio padrão) e das temperaturas do solo. (b) Correlação entre os fluxos de óxido nitroso e as temperaturas do solo

Tabela 1. Emissões de óxido nitroso de solos com cobertura de vegetação nativa na Mata Atlântica e Floresta Amazônica

\begin{tabular}{lccc}
\hline Local & Ecossistema (altitude) & $\begin{array}{c}\text { Fluxo } \\
\left(\mathrm{kg} \mathrm{N} \mathrm{ha}^{-1} \mathrm{ano}^{-1}\right)\end{array}$ & Ref. \\
\hline Mata Atlântica & & & \\
PARNASO (RJ) & FODM (1100-1200 m) & 0,26 & Este estudo \\
PESA (SP) & FODM (1000 m) & 0,8 & 13 \\
PESA (SP) & FODSM (400 m) & 0,9 & 13 \\
PESA (SP) & FODSM (100 m) & 3,6 & 13 \\
RBT (RJ) & FODSM (170-300 m) & 4,2 & 12 \\
PEST (RJ) & FODSM (70-320 m) & $1,0-1,4$ & 14 \\
FNT (RJ) & FOD(SM-M) (500 m) & 2,6 & 14 \\
Coroa Grande (RJ) & FODSM (300-500 m) & 0,9 & 11 \\
Amazônia & & & \\
Bacia Amazônica* & FODSM & $1,4-2,4$ & 24 \\
Bacia Amazônica* & FODSM & $1,1-4,3$ & 25 \\
\hline
\end{tabular}

PARNASO: Parque Nacional da Serra dos Órgãos; PESA: Parque Estadual da Serra do Mar; RBT: Reserva Biológica do Tinguá; PEST: Parque Estadual da Serra da Tiririca; FNT: Floresta Nacional da Tijuca; *Compilação de diversos estudos; FODM: Floresta Ombrófila Densa Montana; FODSM: Floresta Ombrófila Densa Submontana; FOD(SM-M): interface entre FODSM e FODM.

$\mathrm{N}_{2} \mathrm{O}$ na faixa de 0,9 a $4,2 \mathrm{~kg} \mathrm{~N}^{-1} \mathrm{ano}^{-1},{ }^{11-14}$ valores de 3 a 16 vezes superiores à média obtida para a Floresta Ombrófila Densa na Serra dos Órgãos. Estes últimos se assemelham bastante aos fluxos de $\mathrm{N}_{2} \mathrm{O}$ determinados para solos da Floresta Amazônica com cobertura de Floresta Ombrófila Densa Submontana, reportados na faixa 1,1-4,3 $\mathrm{kg} \mathrm{N} \mathrm{ha-1}$ ano $^{-1} \cdot{ }^{24,25}$

\section{CONCLUSÕES}

O fluxo médio de $\mathrm{N}_{2} \mathrm{O}$ em solos com cobertura de Floresta Ombrófila Densa Montana na Serra do Mar (localmente Serra dos Órgãos) foi de aproximadamente $3,0 \mu \mathrm{g} \mathrm{N} \mathrm{m}{ }^{-2} \mathrm{~h}^{-1}\left(0,26 \mathrm{~kg} \mathrm{~N}^{-1}\right.$ $\mathrm{ano}^{-1}$ ) num local onde a precipitação pluviométrica anual se aproxima de $3 \mathrm{~m}$. Os fluxos de $\mathrm{N}_{2} \mathrm{O}$ variaram espacial e sazonalmente, sendo a variação desta última mais acentuada que a primeira. As variações sazonais dos fluxos de $\mathrm{N}_{2} \mathrm{O}$ parecem responder positivamente às variações sazonais de temperatura do solo e de precipitação, entretanto, não é possível se identificar qual desses fatores prevalece no controle da intensidade dos fluxos de $\mathrm{N}_{2} \mathrm{O}$. Os fluxos de $\mathrm{N}_{2} \mathrm{O}$ foram baixos quando comparados aos de solos com cobertura vegetal natural de outras regiões de domínio dos Biomas da Mata Atlântica e da Floresta Amazônica. Outros estudos são necessários para elucidar quais fatores são determinantes no controle da grandeza dos fluxos de $\mathrm{N}_{2} \mathrm{O}$ na área estudada.

\section{MATERIAL SUPLEMENTAR}

A Figura 1S representa a área de estudo - Parque Nacional da Serra dos Órgãos (RJ). Está disponível em http://quimicanova.sbq. org.br, na forma de arquivo PDF, com acesso livre.

\section{AGRADECIMENTOS}

Ao CNPq (Processos 474113/2003-5 e 301421-2009/9), ao FNMA (Projeto "Deposições Atmosféricas" no 1108/94), à CAPES pela bolsa de mestrado de R. A. R. Rodrigues, à toda administração do PARNASO, especialmente E. B. V. de Castro e C. C. de Faria pela valorização e enorme apoio dado às atividades de pesquisas nessa Unidade de Conservação.

\section{REFERÊNCIAS}

1. McElroy, M. B.; The Atmospheric Environment - Effects of Human Activities, Princeton University Press: Princeton, 2002.

2. Crutzen, P. J.; Ambio 1972, 1, 41.

3. http://agage.eas.gatech.edu, acessada em Junho 2012.

4. http://www.metoffice.gov.uk, acessada em Junho 2012.

5. Ravishankara, A. R.; Daniel, J. S.; Portmann, R. W.; Science 2010, 326, 123.

6. IPCC - Intergovernmental Panel on Climate Change; Climate Change 2001: The Scientific Basis: Contribution of Working Group I to the Third Assessment Report of the Intergovernmental Panel on Climate Change, Cambridge University Press: New York, 2001.

7. Goreau, T. J.; de Mello, W. Z. Em Proceedings of the Workshop on Biogeochemistry of Tropical Rain Forests: Problems for Research; Athie, D.; Lovejoy, T.; Oyens, P. M., eds.; Centro de Energia Nuclear na Agricultura e World Wildlife Fund: Piracicaba, 1985.

8. Goreau, T. J.; de Mello, W. Z.; Ambio 1988 17, 275.

9. Santos, C. P. F.; Valles, G. F.; Sestini, M. F.; Hoffman, P.; Dousseau, S. L.; Homem de Mello, A. J.; Anais do XIII Simpósio Brasileiro de Sensoriamento Remoto, Florianópolis, Brasil, 2007.

10. http://www.tedxmataatlantica.com.br/?p=314, acessada em Junho 2012.

11. de Mello, W. Z.; Goreau, T. J.; Geochim. Brasil. 1998, 12, 17.

12. Maddock, J. E. L.; Santos, M. B. P.; Prata, K. R.; J. Geophys. Res. 2001, 106, 23055.

13. Souza Neto, E.; Carmo, J. B.; Keller, M.; Martins, S. C.; Alves, L. F.; Vieira, S. A.; Piccolo, M. C.; Camargo, P.; Couto, Joly, C. A.; Martinelli, L. A.; Biogeosciences 2011, 8, 733.

14. Costa, C. M. P.; Tese de Doutorado, Universidade Federal Fluminense, Brasil, 2002. 
15. IBGE - Instituto Brasileiro de Geografia e Estatística; Manual Técnico de Vegetação Brasileira, Fundação Instituto Brasileiro de Geografia e Estatística: Rio de Janeiro, 1992.

16. Rodrigues, R. A. R.; de Mello, W. Z.; de Souza, P. A. Em Ciência e Conservação na Serra dos Órgãos; Cronemberger, C.; Castro, E. B. V., eds.; Instituto Brasileiro do Meio Ambiente e dos Recursos Naturais Renováveis: Brasília, 2007.

17. Rodrigues, R. A. R.; de Mello, W. Z.; de Souza, P. A.; Quim. Nova 2007, 30,1842

18. Carvalho Junior, O. A.; Coelho, M. A. N.; Martins, E. S.; Gomes, R. A. T.; Couto Junior, A. F.; Oliveira, S. N.; Santana, O. A.; Rev. Bras. Geof. 2008, 26, 331 .

19. FIDERJ - Fundação Instituto de Desenvolvimento Econômico e Social do Rio de Janeiro; Indicadores Climatológicos, Governadoria do Estado do Rio de Janeiro/Secretaria de Planejamento e Coordenação Geral: Rio de Janeiro, 1978.

20. de Mello, W. Z.; Hines, M. E.; J. Geophys. Res. 1994, 99, 14601.

21. Chapuis-Lardy, L.; Wrage, N.; Metay, A.; Chotte, J.-L.; Bernoux, M.; Glob. Change Biol. 2007, 13, 1 .

22. de Souza, P. A., comunicação pessoal.

23. Wrage, N.; Velthof, G. L.; van Beisichem, M. L.; Oenema, O.; Soil Biol. Biochem. 2001, 33, 1723.

24. Davidson, E. A.; Bustamante, M. M. C.; Pinto, A. S.; The Scientific World 2001, 1, 312.

25. Bustamante, M. M. C.; Keller, M.; Silva, D.A. Em Amazonia and Global Change; Keller, M.; Bustamante, M. M. C.; Gash, J.; Dias, P. S., eds.; American Geophysical Union: Washington, 2009. 


\section{FLUXOS DE ÓXIDO NITROSO EM SOLOS COM COBERTURA DE FLORESTA OMBRÓFILA DENSA MONTANA NA SERRA DOS ÓRGÃOS, RIO DE JANEIRO}

Renato de Aragão Ribeiro Rodrigues

Empresa Brasileira de Pesquisa Agropecuária, Embrapa Agrossilvipastoril, CP 343, 78550-970 Sinop - MT, Brasil William Zamboni de Mello*

Departamento de Geoquímica, Instituto de Química, Universidade Federal Fluminense, Outeiro de São João Batista, s/n, 24020-007 Niterói - RJ, Brasil

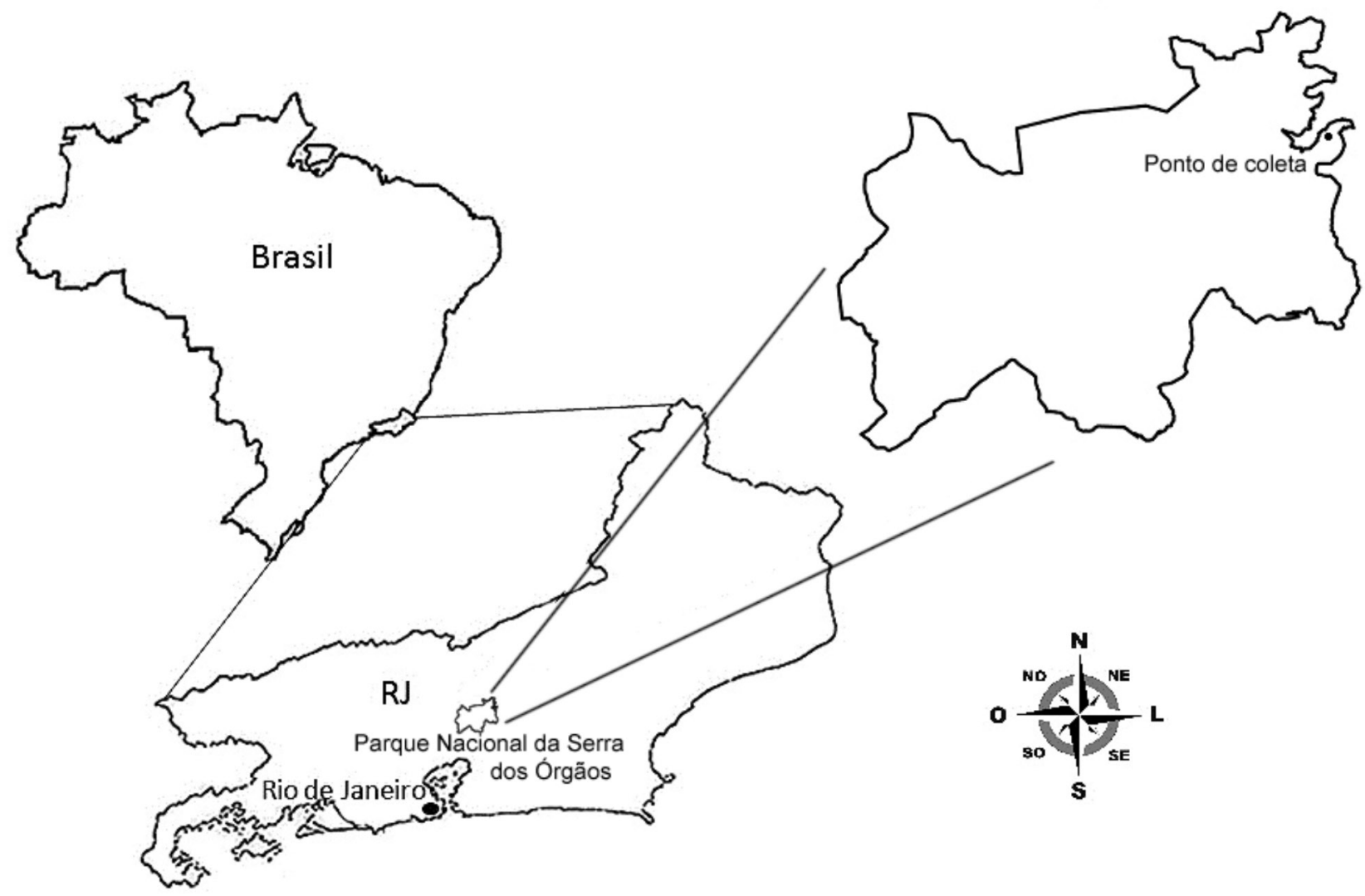

Figura 1S. Área de estudo - Parque Nacional da Serra dos Órgãos (RJ) 